Article

\title{
Photomechanical Response of Composite Structures Built from Azobenzene Liquid Crystal Polymer Networks
}

\author{
Kyung Min Lee ${ }^{1,2}$ and Timothy J. White ${ }^{1, *}$
}

1 Air Force Research Laboratory, Materials and Manufacturing Directorate, Wright Patterson Air Force Base, OH 45433, USA; E-Mail: kyungmin.lee@wpafb.af.mil

2 Azimuth Corporation, Dayton, $\mathrm{OH} 45433$, USA

* Author to whom correspondence should be addressed; E-Mail: timothy.white2@wpafb.af.mil; Tel.: +1-937-255-9551; Fax: +1-937-255-11-282.

Received: 1 August 2011; in revised form: 19 August 2011 / Accepted: 30 August 2011 /

Published: 2 September 2011

\begin{abstract}
Optically directed shape adaptive responses have been sought after for many decades in photoresponsive polymeric materials. A number of recent examinations have elucidated elucidated the unique opportunities of photomechanical responses realized in azobenzene-functionalized liquid crystalline polymer networks (both elastomers and glasses). This work summarizes and contrasts the photomechanical response of glassy polydomain, monodomain, and twisted nematic azo-LCN materials to blue-green irradiation. Building from this summary, the combinatorial photomechanical response observed upon irradiation of composite cantilevers is examined. Large scale shape adaptations are realized, with novel responses that may be of potential use in future employment of these materials in actuation.
\end{abstract}

Keywords: azobenzene; liquid crystal; photomechanical

\section{Introduction}

Composites blend the distinctive properties of component materials to tailor performance towards a practical goal. For example, high strength polymeric materials such as Kevlar are blended with conventional polymeric materials as well as other fibers to yield soft, flexible bullet-proof armor that trades off strength for cost and wear-ability. Mechanical systems have extensively employed 
composites for a variety of functional purposes. Grading material properties along the length or through the thickness of a cantilever is a mature and well-developed community due to its extensive relevance and employment in architectural applications.

The focus of this work is the development of distinctive photomechanical responses in composite structures fabricated from photoresponsive polymeric materials. Photomechanical effects in polymeric materials have been examined by researchers for more than four decades [1]. Photochromic molecules (primarily azobenzene, although other molecules have been employed [2]) predominately are covalently bonded into the polymer network but at times have been added as a guest molecule $[3,4]$. The photomechanical response of these materials is strongly dictated by the structure of the polymer network. Early work focused on amorphous and semi-crystalline polymers, with maximal observed photogenerated strain cycles of only 1\% [5]. Work emanating from Finkelmann et al. considerably expanded the capability of generating larger magnitude strain cycles $(\sim 20 \%)$ by fabricating azobenzene-functionalized liquid crystal elastomers (azo-LCE) [6]. Since this work, considerable effort has pursued a variety of aspects of this unique means of transducing energy in primarily liquid crystalline materials. Notably, this research has expanded into looking at glassy, azobenzene-functionalized liquid crystal polymers [7-16]. To capture both elastomeric and glassy liquid crystalline systems, we refer to the broader area as azobenzene-functionalized liquid crystal polymer networks (azo-LCN) and emphasize the glassy or elastomeric nature of the given chemistry.

Photomechanical responses in azo-LCN materials have been most often observed as uniaxial contraction of a film (typically elastomers - large strain) [6,17-19] or bending of a cantilever (typically glasses - lower strain) [7-16]. The magnitude or directionality of the photomechanical response of azo-LCN materials has repeatedly been controlled by the alignment of the linear polarization of the actinic source and the sample geometry $[8,9,11,12,14-16]$. Theoreticians have found the unique blend of polymer physics, photochemistry, and mechanics a rich challenge [18,20-22].

A recent theme in the literature is the employment of LCN materials to generate three-dimensional mechanical responses. A consortium of international researchers recently presented an elegant experimental and theoretical study the observation of thermally-directed coiling in twisted nematic elastomeric LCN materials [23]. In addition to a recent report on optically-directed flexural-torsional oscillations [10] we have also observed similar, photochemically/photothermally induced coiling in azo-LCN materials [24]. Warner has presented theoretical descriptions of twisted nematic systems [25] as well as spatially defined defect structures [26] that potentially allow for complex responses which when realized experimentally, will yield a variety of unconventional and potentially useful mechanical responses in these materials.

Relevant to the work presented here; Ikeda, Broer, and Yu have recently published examples of the functionality enabled by embedding azo-LCN materials within other polymeric materials. Ikeda et al. demonstrated photodriven motor and inchworm like behavior [27-29] enabled by laminating the azo-LCN materials in conventional polymeric materials. Broer wonderfully leveraged the ability to form intricate structures via inkjet printing onto polymeric media to generate active, photoresponsive cilia [30]. Yu and coworkers have developed photodirected robotic elements, with azo-LCN materials laminated in polyethylene [31].

In this work, we begin by summarizing the unique photomechanical responses that we have observed in glassy azo-LCN materials of a variety of domain structures and orientations. Building 
from this summary, we demonstrate unique photomechanical responses observed when these materials are blended into composite structures. While not particularly tailored to any use or application, the fabrication of composite structures presented here is another approach to realize complex shape adaptations necessary for advanced applications.

\section{Experimental Section}

Azobenzene liquid crystal polymer networks of a variety of domain structures and orientations were prepared as specified in previous reports (monodomain [10,14], polydomain [8,9], twisted nematic [24], polyimide [32]). In general, a mixture composed of $20 \mathrm{wt} \%$ 4-4'-bis[6-(acryoloxy)hexyloxy]-azobenzene (2azo, BEAM Engineering for Advanced Measurements) was mixed with RM257 (Merck) (chemical structures shown in Figure 1). Approximately $1 \mathrm{wt} \%$ Irgacure 784 (I-784) was added to this mixture to enable visible light photopolymerization induced by exposure to the expanded beam of a $532 \mathrm{~nm}$ $\mathrm{Nd}$ :YAG CW laser. The mixture was subsequently heated to approximately $150{ }^{\circ} \mathrm{C}$ and stirred to melt and homogenize the solid materials. The mixture was then drawn into liquid crystal alignment cells by capillary action at approximately $100{ }^{\circ} \mathrm{C}$. The domain structure and orientation were controlled by the use of rubbing and its directionality. Polydomain azo-LCN (PD-azo-LCN) were fabricated by curing at $130{ }^{\circ} \mathrm{C}$ in alignment cells with un-rubbed Elvamide. Monodomain azo-LCN (MD-azo-LCN) were fabricated by curing at $75^{\circ} \mathrm{C}$ in alignment cells with anti-parallel rubbed Elvamide layers. Twisted nematic azo-LCN (TN-azo-LCN) were fabricated in a similar fashion to MD-azo-LCN but the rubbing direction of the alignment layers was orthogonal to one another. Polymerization times varied, but typically were on the order of 15-30 min. After polymerization, the liquid crystal alignment cells were opened and the material was extracted with a razor blade and the rubbing material was removed from the films by a methanol wash. In all cases, upon polymerization the azo-LCN material exhibited a glass transition temperature above room temperature typically on the order of $45-60{ }^{\circ} \mathrm{C}$. Composites of PD-azo-LCN, MD-azo-LCN, and TN-azo-LCN were fabricated by cutting pieces of the films and gluing together with Norland optical adhesive (NOA65). Unless otherwise noted, sample thickness was approximately $15 \mu \mathrm{m}$.

Figure 1. (a) Chemical structures of monomers employed here. The approximate concentration of 2 azo was $20 \mathrm{wt} \%$. (b) Illustration of cantilever geometry and terminology. $\mathrm{E}$ is used to denote the electric field vector of the polarization of light.

a

RM257

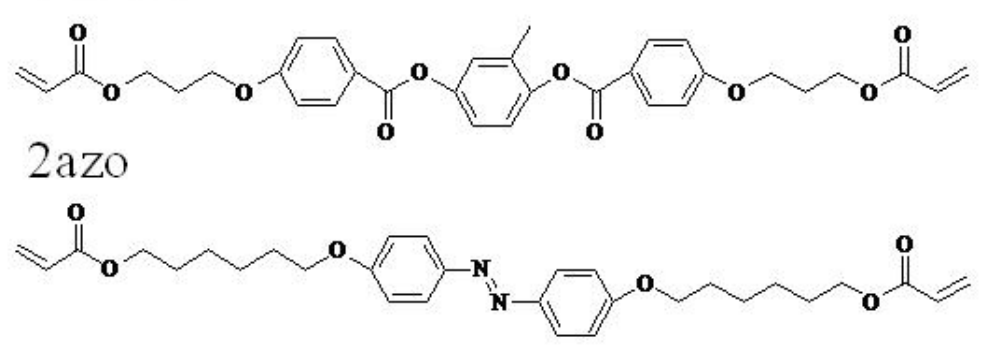

b

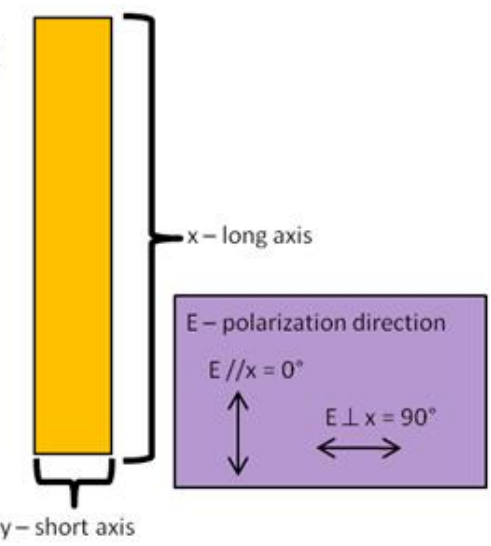


The photomechanical responses presented here were induced by exposure to the $442 \mathrm{~nm}$ line of a Helium-Cadmium ( $\mathrm{HeCd}$ ) laser at intensities ranging from $80-850 \mathrm{~mW} / \mathrm{cm}^{2}$ (specified accordingly). Images were captured with a charge coupled device (CCD) operating at $30 \mathrm{~Hz}$. The domain orientation and alignment presented here was captured with a polarized optical microscope (POM).

\section{Results and Discussion}

Photomechanical responses in azobenzene-functionalized polymeric materials have nearly exclusively utilized UV light. UV light is well-known to efficiently and nearly completely isomerize azobenzene from trans to cis. In amorphous and semi-crystalline materials, the change in the molecular length from trans $(9 \AA)$ to cis $(5.5 \AA)$ imparts local stress on the network and is the source of the mechanical response. The comparably amplified responses observed in elastomeric liquid crystalline polymer networks (azo-LCN) are enabled by the association of liquid crystalline order and the configurational state of azobenzene, as the rod-like trans isomer is order-favorable while the bent cis isomer is order-disrupting.

Comparatively our previous work has leveraged so-called blue-green light $(440-514 \mathrm{~nm})$ that is able to induce both trans-cis and cis-trans isomerization of azobenzene. When linearly polarized, light in this wavelength regime has been shown to reorient azobenzene chromophores normal to electric field vector of the incident photons yielding volume and surface relief gratings in azobenzene polymers [33]. The reorientation of the azobenzene chomophores occurs due to the nearly equivalent absorbance of the azobenzene isomers, which correspondingly cycle the chromophores between the two states. Because of the dichroic absorbance of the azobenzene isomers, trans and cis azobenzene statistically build up oriented normal to the polarization of the incident irradiation-as this is the lowest absorption condition for the chromophores. The observation of optically-fixed shape memory [9] in these materials is due to the glassy nature of the materials, which prevents the chromophores from returning to the original alignment. Exposure to circularly polarized light or heating past the glass transition temperature returns the material to the original state.

The manifestation of this photochemical mechanism in glassy azo-LCN materials is summarized in Figure 2 for PD-azo-LCN, MD-azo-LCN, and TN-azo-LCN. Each of these samples was prepared with the formulation documented in Figure 1, based on a crosslinking azobenzene mesogenic monomer referred to here as 2azo. As first reported in 2005, exposure of PD-azo-LCN materials allows for bidirectional bending upon irradiation with blue-green light [8,11]. For the sample presented in Figure 2, $442 \mathrm{~nm}$ light is strongly attenuated through the thickness of the cantilever and accordingly, the photogenerated strain is nonuniform across the thickness. The orientation of the linear polarization to the cantilever geometry dictates whether the exposed surface of PD-azo-LCN undergoes a contractile (when the polarization ' $\mathrm{E}$ ' is parallel to the long axis of the cantilever ' $\mathrm{x}$ ', $\mathrm{E} / / \mathrm{x}$ ) or expansive $(E \perp x)$ strain. Correspondingly, the directionality of the bending is towards the actinic source when $\mathrm{E} / / \mathrm{x}$ (contraction on front surface) and away from the actinic source when $\mathrm{E} \perp \mathrm{x}$ (expansion on the front surface). To intermediate polarization conditions, less bending is observed as well as some evidence of twisting [8,11]. Although not shown in Figure 2(a), upon removal of $442 \mathrm{~nm}$ the cantilevers retain their bent shape many months [9]. 
Figure 2. (a) Summary of photomechanical response of PD-azo-LCN to irradiation to blue-green light as a function of polarization ( $\mathrm{i}-\mathrm{E} / / \mathrm{x}\left(0^{\circ}\right)$, ii $-\mathrm{E} 15^{\circ}$ to $\mathrm{x}$, iii $-\mathrm{E} 45^{\circ}$ to $\mathrm{x}$, iv- $\mathrm{E} 75^{\circ}$ to $\mathrm{x}, \mathrm{v}-\mathrm{E} 90^{\circ}$ to $\mathrm{x}$ ). (b) Summary of photomechanical response of MD-azo-LCN cantilevers to blue-green light (images vi-xv). The magnitude and directionality of the bending response is dictated by light intensity and the alignment of the liquid crystalline director to the cantilever geometry. (c) Summary of photomechanical response of TN-azo-LCN cantilevers to blue-green light. In all cases, the approximately $15 \mu \mathrm{m}$ cantilevers were exposed to $150 \mathrm{~mW} / \mathrm{cm}^{2} 442 \mathrm{~nm}$ light polarized $\mathrm{E} / / \mathrm{x}$.

(a)

PD-azo-LCN

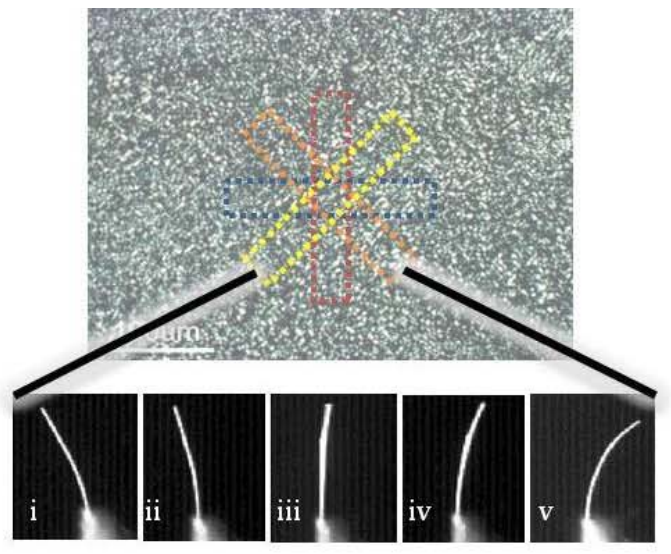

(b)
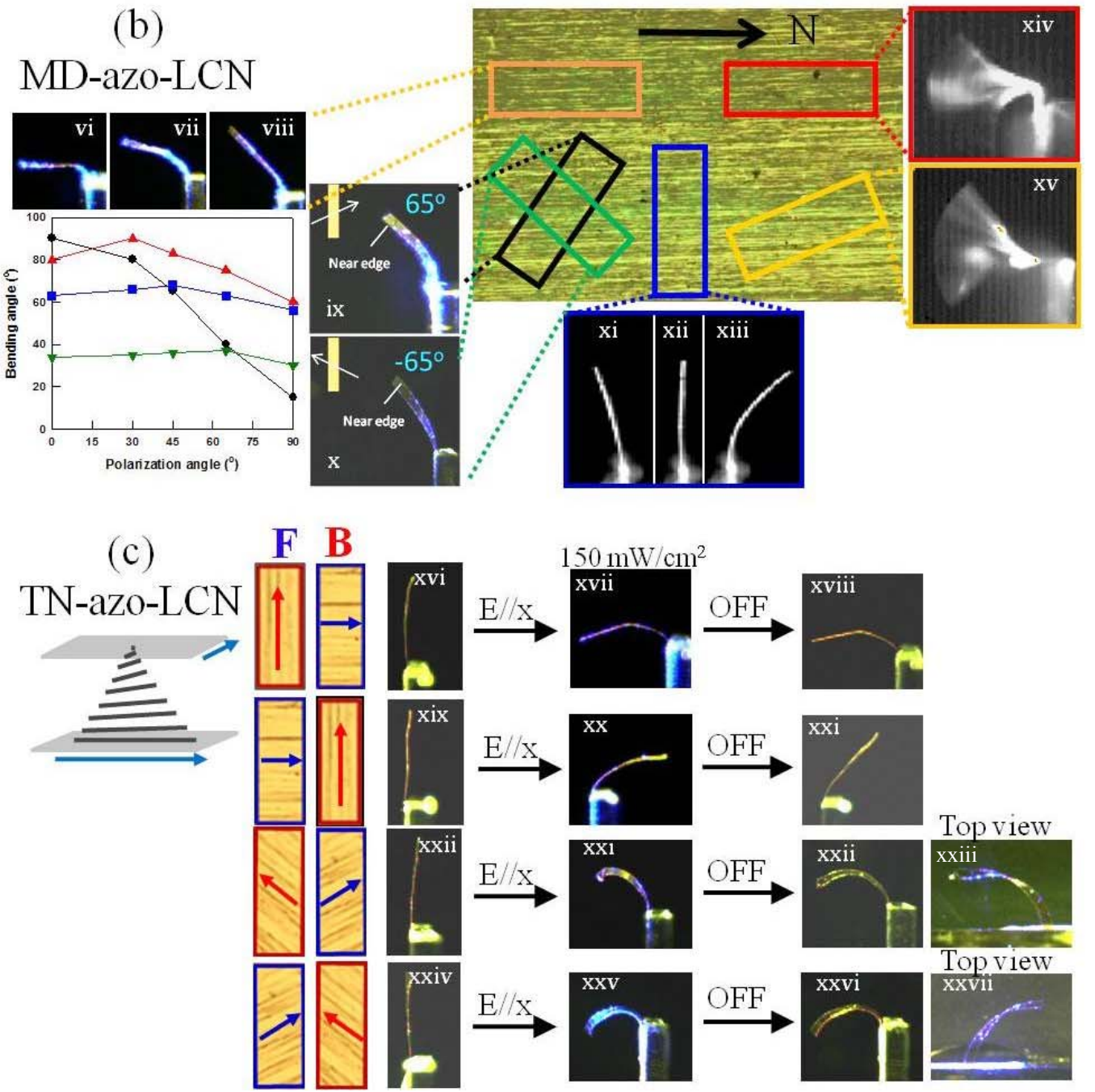

$50 \mathrm{~mW} / \mathrm{cm}^{2}$
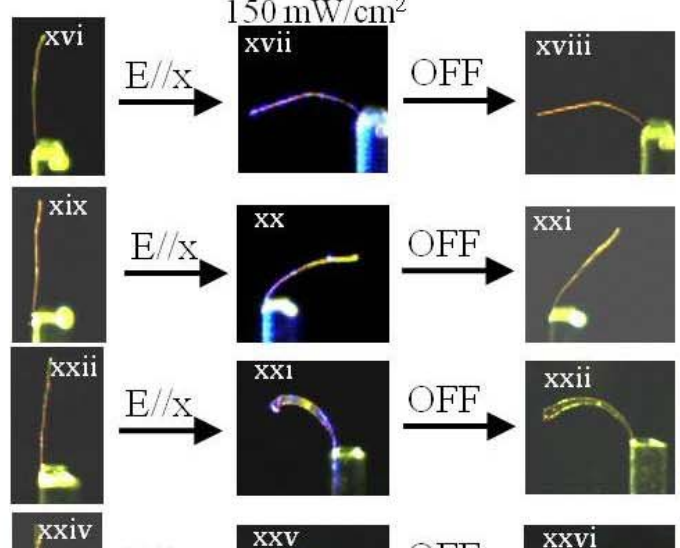

Top view
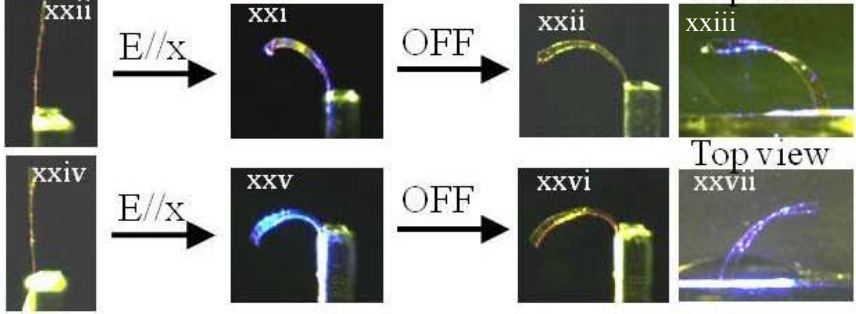

XXV11 
Comparably, MD-azo-LCN materials exhibit more convoluted photomechanical responses, illustrated in Figure 2(b) images vi-xv. Apparent in the inset polarized optical micrograph (POM) is the alignment of the material. The resulting mechanical output is highly dependent on the alignment of the director to the cantilever geometry. When the nematic director of the MD-azo-LCN material is aligned along $\mathrm{x}$, only unidirectional bending is observed (Figure 2(b)-vi-viii). Intuitively, the unidirectional response is not expected if the underlying photochemical mechanism is due to reorientation of the azobenzene chromophores. However, it should be remembered that in MD-azo-LCN materials the initial alignment of the azobenzene chromphores (along $\mathrm{N}$ ) is parallel to $\mathrm{x}$. As such, exposure to $\mathrm{E} / / \mathrm{x}$ is capable of reorienting a considerable portion of the azobenzene mesogens to the short axis of the cantilever (y). However, exposure to $\mathrm{E} \perp \mathrm{x}$ may serve to only improve the alignment of the azobenzene mesogens along $\mathrm{N}$, which in this case is along $\mathrm{x}$. As such, minimal expansive strain is generated and bending is dominated by the generation small concentration of cis azobenzene (est. 2-5\%) that is generated in the photostationary state with $442 \mathrm{~nm}$ exposure. Further complicating the photomechanical response is the geometry of the cantilever (in this case $5 \mathrm{~mm} \times 1 \mathrm{~mm}$ ), which can bias the observed response. Comparatively, when the director of the MD-azo-LCN is aligned such that it is along y, asymmetric bidirectional bending is observed (Figure 2(b)-xi-xiii). In this case, exposure to $\mathrm{E} / \mathrm{x}$ results in slight bending towards the actinic source while exposure to $\mathrm{E} \perp \mathrm{x}$ results in larger bending away from the actinic source. The reversal in the orientation of the director to the principal axes of the cantilever causes a contractile force to $\mathrm{E} / \mathrm{x}$ (due to slight rotation of chromophores along $\mathrm{x}$ to $\mathrm{y}$ ) and an expansive strain to $\mathrm{E} \perp \mathrm{x}$ (due to rotation of chomophores from $\mathrm{y}$ to $\mathrm{x}$ ). When the nematic director is cut at intermediate angles to the principal axes of the o-MD-azo- $\mathrm{LCN}\left( \pm 65^{\circ}\right)$, shear is observed in addition to strain (as illustrated in the images in Figure 2(b)-ix-x as well as inset graph). Focusing the actinic light onto the base of the cantilever has allowed for the observation of high frequency, large amplitude oscillations presented in Figure 2(b)-xiv and Figure 2(b)-xv. When the nematic director of the MD-azo-LCN is parallel to $\mathrm{x}$, the oscillations are in plane (Figure 2(b)-xiv). However, when the nematic director of the MD-azo-LCN are offset between $\mathrm{x}$ and $\mathrm{y}$, the oscillations are flexural-torsional in nature and results in both in plane and out of plane deformations (Figure 2(b)-xv).

Finally, TN-azo-LCN materials exhibit yet another set of photomechanical responses to $442 \mathrm{~nm}$ irradiation. A twisted nematic orientation results in a $90^{\circ}$ shift in orientation of the director through the thickness. We refer to the case where the director on the front surface is parallel to $\mathrm{x}$ as 'case A' and the case where the director on the front surface is normal to $\mathrm{x}$ as 'case B'. As shown in Figure 2(c)-xvii, exposure of TN-azo-LCN to $442 \mathrm{~nm}$ light yields comparatively larger amplitude bending. In the case A TN geometry, incident irradiation on the front surface yields a contraction resulting in bending towards the exposing direction. Because of the TN geometry, $442 \mathrm{~nm}$ light penetrates further into the sample and accordingly, the back surface, which sees appreciable light, undergoes an expansion. Together, the front surface contraction and the back surface expansion cooperate to yield comparably larger magnitude bending. This was first discussed by Broer and coworkers [13] and recently treated theoretically by Warner and coworkers [25]. Reversing the orientation to 'case B' results in large amplitude bending away from the source (Figure 2(c)-xx). The polarization dependence of the responses of case $\mathrm{A}$ and case $\mathrm{B}$ are complicated and a product of irradiation conditions, thickness, and cantilever aspect ratio. In a similar fashion to the offset MD-azo-LCN (o-MD-azo-LCN) when TN-azo-LCN materials are cut such the alignment on the front surface as at an intermediate angle to 
the principal axes of the cantilever, as shown in Figure 2(c)-xxiv, large amplitude twisting is observed. As with MD-azo-LCN cut with the nematic director offset to the principal axes of the cantilever, the comparably larger magnitude twisting is enabled by a shear gradient that once again is cooperative through the thickness.

The photomechanical responses observed in these materials lay the foundation for the remaining portion of this work that focuses on the generation of combintorial photomechanical responses in composites formed from two different azo-LCN materials. In all cases, the azo-LCN materials were glued together with an optical adhesive with great care to assure as close to ideal consistency between the two materials. In a first exercise, the large scale bending observed in MD-azo-LCN is combined with the twisting observed in MD-azo-LCN cut with the nematic director aligned at intermediate angle to the cantilever geometry (o-MD-azo-LCN). Two different o-MD/MD-azo-LCN composite structures are examined in Figure 3 - one where the top portion of the composite cantilever (' $a$ ') is larger than the bottom portion of the composite cantilever (' $b$ ') and another where b is greater than a. In Figure 3(a)-i, to $500 \mathrm{~mW} / \mathrm{cm}^{2}$ of $442 \mathrm{~nm}$ irradiation, the large magnitude bending with slight twisting near the tip of the o-MD/MD-azo-LCN composite cantilever is observed. When subjected to $850 \mathrm{~mW} / \mathrm{cm}^{2} 442 \mathrm{~nm}$ irradiation, the composite cantilever oscillates at low amplitude but with significant rocking/twisting motion (Figure 3(a)-ii). Comparatively, when the o-MD/MD-azo-LCN composite cantilever is composed such that $b$ is greater than $a$, lower magnitude bending is observed upon irradiation with $500 \mathrm{~mW} / \mathrm{cm}^{2}$ of $442 \mathrm{~nm}$ light, but interestingly, similar magnitude twisting. However, when exposed to $850 \mathrm{~mW} / \mathrm{cm}^{2}$ of $442 \mathrm{~nm}$ light, large amplitude high frequency oscillation of the o-MD/MD-azo-LCN composite cantilever is realized. Although difficult to visualize in a static image, the oscillation motion is exclusively in plane near the base of the cantilever. However, out of plane twisting motion is observed when the cantilever traverses through the focused laser beam. This type of effect varies from the recently presented flexural-torsional oscillation of these materials [10] in that the twisting is localized to the upper portion of the cantilever.

Figure 3. Photomechanical response of a composite cantilevers composed of a MD-azo-LCN base ("a") and an offset MD-azo-LCN tip (o-MD-azo-LCN) ("b") observed upon irradiation to 500 and $850 \mathrm{~mW} / \mathrm{cm}^{2}$ in the condition when (a) a $>$ b and (b) a $<$ b.

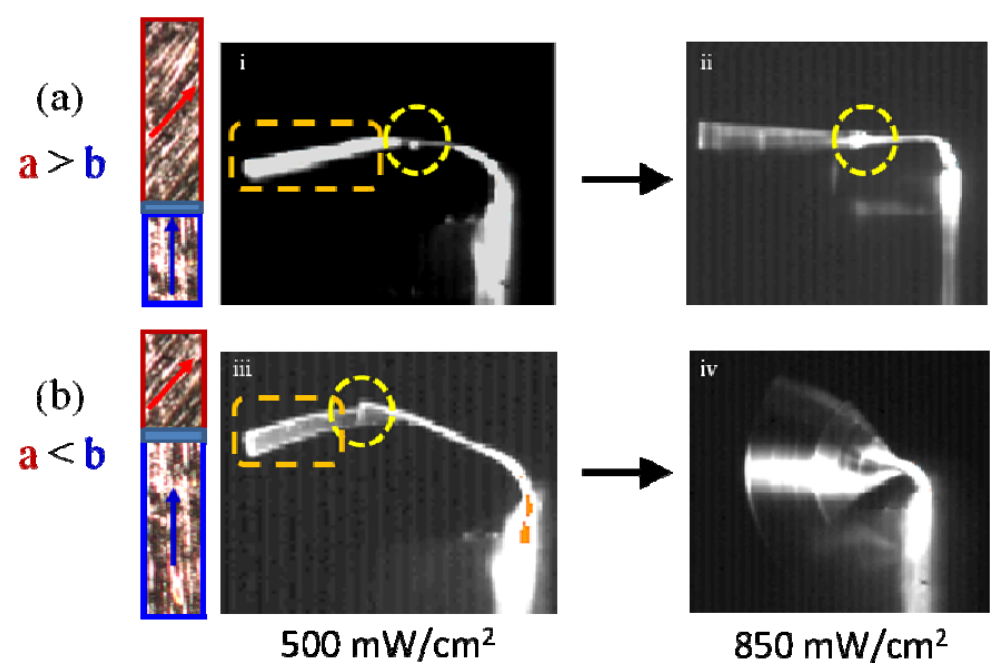


Recently, large scale photomechanical responses were reported in azobenzene-functionalized polyimide materials with glass transition temperatures exceeding $200{ }^{\circ} \mathrm{C}$. These high performance polymers offer comparably larger moduli which more efficiently translates light into work. However, the photoinduced bending of these materials is comparably slower than that observed in azo-LCN materials and limited to static deformation. For comparison, the deformation of the azo-PI cantilever is shown in Figure 4(a)-i-iii. Upon exposure to $442 \mathrm{~nm}$ polarized E//x, the cantilever bends towards the laser to $500 \mathrm{~mW} / \mathrm{cm}^{2}$ and $850 \mathrm{~mW} / \mathrm{cm}^{2}$. Increasing laser intensity slightly increases the bending angle. Bending occurs over the course of $30 \mathrm{~min}$. Similar to Figure 3, two azo-PI/MD-azo-LCN composite structures were examined. In Figure 4(b)-iv-vi, the MD-azo-LCN portion is less than $50 \%$ of the length of the total composite $(5 \mathrm{~mm})$. Upon irradiation to $500 \mathrm{~mW} / \mathrm{cm}^{2}$, larger angle and comparably faster bending is observed. Bending to this maximum angle occurs within $30 \mathrm{~s}$. Subjecting the composite cantilever to $850 \mathrm{~mW} / \mathrm{cm}^{2}$ induces low amplitude oscillation. Increasing the length of the MD-azo-LCN portion of the cantilever serves to increase the magnitude of the bending and amplitude of the oscillation (Figure 4(c)-vii-ix).

Figure 4. Photomechanical response of a azo-PI and azo-PI composite cantilevers composed of a MD-azo-LCN base ("a") and the glassy azo-PI material (azo-PI) ("b") observed upon irradiation to 500 and $850 \mathrm{~mW} / \mathrm{cm}^{2}$. (a) Azo-PI monolithic cantilever, (b) a $<$ b, and (c) a $>$ b.

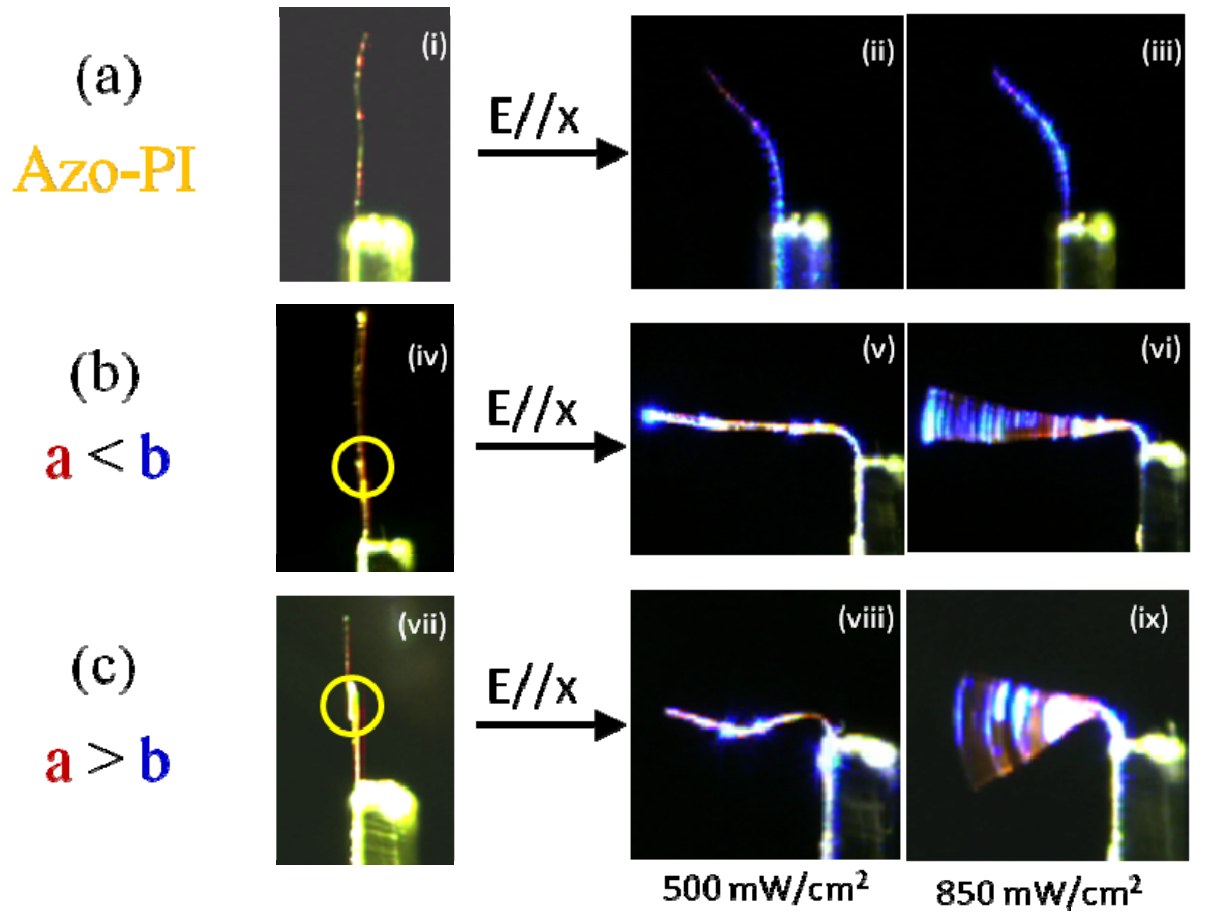

The unique opportunity of spatially directing distinctive photomechanical responses is demonstrated in Figure 5, where a composite of a PD-azo-LCN cantilever with a TN-azo-LCN film attached to it at a singular point. Exposure of the cantilever base allows the composite structure to be deflected towards or away from the laser source, dictated by the typical response of the PD-azo-LCN material. As previously discussed, PD-azo-LCN materials can retain their shape upon removal of the irradiation $[8,9]$. Subsequent exposure of the TN-azo-LCN portion of the material can open and close the appending 
structure. Conceivably, the opening/closing of the TN-azo-LCN could act as a wirelessrobotic element. Video of the actuation of this composite structure is included in the supporting material.

Figure 5. Photomechanical response of a TN-azo-LCN/PD-azo-LCN composite structure. When the PD-azo-LCN base is irradiation with $100 \mathrm{~mW} / \mathrm{cm}^{2}$, optically fixable forward/reverse bending is observed. Addressing the TN-azo-LCN portion of the structure opens and closes the "mouth" of structure. See MOVIE1 in Support Information.

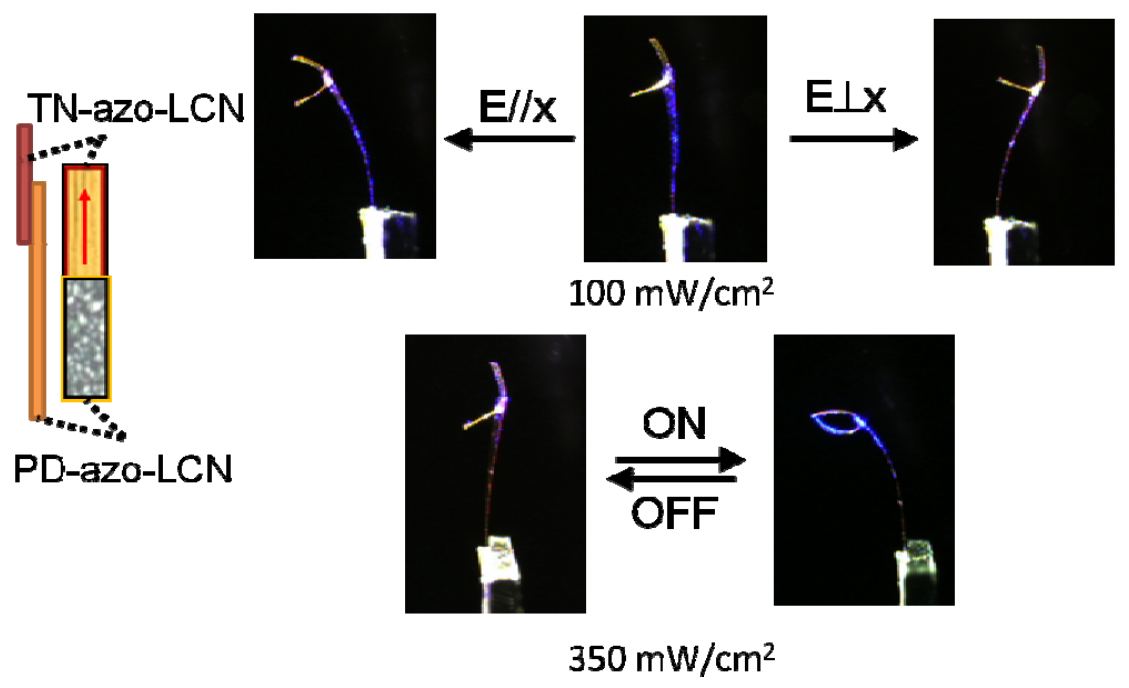

\section{Conclusions}

In summary, based on the unique photomechanical effects attainable observed upon blue-green irradiation of glassy azo-LCN materials, novel combinatorial responses are realized in composite structures. Three composites were presented in this work. First, static and oscillatory deformation of MD-azo-LCN with spatially varying response composed of planar deflection at the cantilever base and twisting at the tip were observed when combined with an offset-MD-azo-LCN material. Second, the rapid and oscillatory deformation of MD-azo-LCN materials was combined with the thermal stability and strength of azobenzene-functionalized polyimides. Combining speed with strength in a composite could more efficiently tranduce light into work in these materials. Finally, distinctive mechanical responses were separately cued by the selective, spatial regulation of light exposure of a TN-azoLCN/PD-azo-LCN composite. The composite structure was bent forward and reversed, optically fixed with the blue-green irradiation. Subsequently, the "mouth" of the structure (e.g., the TN-azo-LCN) could be opened and closed.

\section{Acknowledgements}

We are grateful to the Air Force Office of Scientific Research and the Materials and Manufacturing Directorate of the Air Force Research Laboratory for funding. Sincere appreciation to David Wang and Loon-Seng Tan for the synthesis of the azobenzene-functionalized polyimide. We acknowledge insightful discussions with our colleagues Timothy Bunning, Richard Vaia, Hilmar Koerner, Matthew Smith, and Nelson Tabiryan. 


\section{References}

1. Lovrien, R. The photoviscosity effect. Proc. Natl. Acad. Sci. USA 1967, 57, 236-242.

2. Athanassiou, A.; Kalyva, M.; Lakiotaki, K.; Georgiou, S.; Fotakis, C. All-optical reversible actuation of photochromic-polymer microsystems. Adv. Mater. 2005, 17, 988-992.

3. Camacho-Lopez, M.; Finkelmann, H.; Palffy-Muhoray, P.; Shelley, M. Fast liquid crystal elastomer swims in the dark. Nat. Mater. 2004, 3, 307-310.

4. Cojocariu, C.; Rochon, P. Light-induced motions in azobenzene-containing polymers. Pure Appl. Chem. 2004, 76, 1479-1497.

5. Eisenbach, C.D. Isomerization of aromatic azo chromophores in poly(ethyl acrylate) networks and photomechanical effect. Polymer 1980, 21, 1175-1179.

6. Finkelmann, H.; Nishikawa, E.; Pereira, G.G.; Warner, M. A new opto-mechanical effect in solids. Phys. Rev. Lett. 2001, 87, 015501.

7. Harris, K.D.; Cuypers, R.; Scheibe, P.; van Oosten, C.L.; Bastiaansen, C.W.M.; Lub, J.; Broer, D.J. Large amplitude light-induced motion in high elastic modulus polymer actuators. J. Mater. Chem. 2005, 15, 5043-5048.

8. Lee, K.M.; Koerner, H.; Vaia, R.A.; Bunning, T.J.; White, T.J. Relationship between the photomechanical response and the thermomechanical properties of azobenzene liquid crystalline polymer networks. Macromolecules 2010, 43, 8185-8190.

9. Lee, K.M.; Koerner, H.; Vaia, R.A.; Bunning, T.J.; White, T.J. Light-activated shape memory of glassy azobenzene liquid crystal polymer networks. Soft Matter 2011, 7, 4318-4324.

10. Lee, K.M.; Smith, M.L.; Koerner, H.; Tabiryan, N.; Vaia, R.A.; Bunning, T.J.; White, T.J. Photodriven, flexural-torsional oscillations of glassy azobenzene liquid crystal polymer networks. Adv. Funct. Mater. 2011, doi: 10.1002/adfm.201100333.

11. Tabiryan, N.; Serak, S.; Dai, X.-M.; Bunning, T. Polymer film with optically controlled form and actuation. Opt. Express 2005, 13, 7442-7448.

12. van Oosten, C.L.; Corbett, D.; Davies, D.; Warner, M.; Bastiaansen, C.W.M.; Broer, D.J. Bending dynamics and directionality reversal in liquid crystal network photoactuators. Macromolecules 2008, 41, 8592-8596.

13. van Oosten, C.L.; Harris, K.D.; Bastiaansen, C.W.M.; Broer, D.J. Glassy photomechanical liquid-crystal network actuators for microscale devices. Eur. Phys. J. E 2007, 23, 329-336.

14. White, T.J.; Serak, S.V.; Tabiryan, N.V.; Vaia, R.A.; Bunning, T.J. Polarization-controlled, photodriven bending in monodomain liquid crystal elastomer cantilevers. J. Mater. Chem. 2009, 19, 1080-1085.

15. White, T.J.; Tabiryan, N.; Tondiglia, V.P.; Serak, S.; Hrozhyk, U.; Vaia, R.A.; Bunning, T.J. High frequency photodriven polymer oscillator. Soft Matter 2008, 4, 1796-1798.

16. Yu, Y.; Nakano, M.; Ikeda, T. Photomechanics: Directed bending of a polymer film by light. Nature 2003, 425, 145.

17. Cviklinski, J.; Tajbakhsh, A.R.; Terentjev, E.M. UV isomerisation in nematic elastomers as a route to photo-mechanical transducer. Eur. Phys. J. E 2002, 9, 427-434.

18. Hogan, P.M.; Tajbakhsh, A.R.; Terentjev, E.M. UV manipulation of order and macroscopic shape in nematic elastomers. Phys. Rev. E: Stat. Nonlinear Soft Matter Phys. 2002, 65, 041720. 
19. Li, M.-H.; Keller, P.; Li, B.; Wang, X.; Brunet, M. Light-driven side-on nematic elastomer actuators. Adv. Mater. 2003, 15, 569-572.

20. Corbett, D.; Warner, M. Nonlinear photoresponse of disordered elastomers. Phys. Rev. Lett. 2006, 96, 237802.

21. Corbett, D.; Warner, M. Bleaching and stimulated recovery of dyes and of photocantilevers. Phys. Rev. E: Stat. Nonlinear Soft Matter Phys. 2008, 77, 051710/051711-051710/051711.

22. Warner, M.; Mahadevan, L. Photoinduced deformations of beams, plates, and films. Phys. Rev. Lett. 2004, 92, 134302.

23. Sawa, Y.; Ye, F.; Urayama, K.; Takigawa, T.; Gimenez-Pinto, V.; Selinger, R.L.B.; Selinger, J.V. Shape selection of twist-nematic-elastomer ribbons. Proc. Natl. Acad. Sci. USA 2011, 108, 6364-6368.

24. Lee, K.M.; Smith, M.L.; Vaia, R.A.; Bunning, T.; White, T.J. Large magnitude, optically directed coiling observable in twisted nematic azobenzene liquid crystal polymer networks. 2011, unpublished.

25. Modes, C.D.; Warner, M.; van Oosten, C.L.; Corbett, D. Anisotropic response of glassy splay-bend and twist nematic cantilevers to light and heat. Phys. Rev. E: Stat. Nonlinear Soft Matter Phys. 2010, 82, 041111.

26. Modes, C.D.; Bhattacharya, K.; Warner, M. Disclination-mediated thermo-optical response in nematic glass sheets. Phys. Rev. E 2010, 81, 060701.

27. Naka, Y.; Mamiya, J.-I.; Shishido, A.; Washio, M.; Ikeda, T. Direct fabrication of photomobile polymer materials with an adhesive-free bilayer structure by electron-beam irradiation. J. Mater. Chem. 2011, 21, 1681-1683.

28. Yamada, M.; Kondo, M.; Mamiya, J.-I.; Yu, Y.; Kinoshita, M.; Barrett, C.J.; Ikeda, T. Photomobile polymer materials: towards light-driven plastic motors. Angew. Chem. Int. Ed. 2008, 47, 4986-4988.

29. Yamada, M.; Kondo, M.; Miyasato, R.; Naka, Y.; Mamiya, J.-I.; Kinoshita, M.; Shishido, A.; Yu, Y.; Barrett, C.J.; Ikeda, T. Photomobile polymer materials-various three-dimensional movements. J. Mater. Chem. 2009, 19, 60-62.

30. van Oosten, C.L.; Bastiaansen, C.W.M.; Broer, D.J. Printed artificial cilia from liquid-crystal network actuators modularly driven by light. Nat. Mater. 2009, 8, 677-682.

31. Cheng, F.; Yin, R.; Zhang, Y.; Yen, C.-C.; Yu, Y. Fully plastic microrobots which manipulate objects using only visible light. Soft Matter 2010, 6, 3447-3449.

32. Wang, D.H.; Lee, K.M.; Yu, Z.; Koerner, H.; Vaia, R.A.; White, T.J.; Tan, L.-S. Photomechanical response of glassy azobenzene polyimide networks. Macromolecules 2011, 44, 3840-3846.

33. Viswanathan, N.K.; Kim, D.U.; Bian, S.; Williams, J.; Liu, W.; Li, L.; Samuelson, L.; Kumar, J.; Tripathy, S.K. Surface relief structures on azo polymer films. J. Mater. Chem. 1999, 9, 1941-1955.

(C) 2011 by the authors; licensee MDPI, Basel, Switzerland. This article is an open access article distributed under the terms and conditions of the Creative Commons Attribution license (http://creativecommons.org/licenses/by/3.0/). 